\title{
The Transillumination Possibility of Imidazole-Osmium Postfixed Tissue and Its Consequences for the Handling of Tissue Samples
}

\author{
Tilman Voigt ${ }^{1, *}$ and Wolfgang Dauber ${ }^{2}$ \\ ${ }^{1}$ Institute of Anatomy, University of Fribourg, Rte. Albert-Gockel 1, CH-1700 Fribourg, Switzerland \\ ${ }^{2}$ Institute of Anatomy, Eberhard-Karls-University Tuebingen, 72074 Tuebingen, Germany
}

\begin{abstract}
Osmium postfixation is established as a routine procedure for transmission electron microscopy (TEM). On the one hand, this routine procedure leads to good results for TEM, but on the other hand results in blackened tissue samples that do not allow examination of any structures within the embedded tissue sample by a light microscope. Equivalent fixation results for TEM are achieved with imidazole-osmium postfixation, and with this postfixation method tissue is not blackened and can be transilluminated with point light sources. This allows easier recognition of histological details within tissue samples and makes it possible to screen embedded samples for appropriate ultrastructural processing. Jejunum is used to demonstrate the method.
\end{abstract}

Key words: imidazole, osmium, fixation, technique, electron microscopy, light microscopy, jejunum

\section{INTRODUCTION}

Osmium postfixation is established as a routine method for tissue preparation for transmission electron microscopy (TEM). A disadvantage of this postfixation is that osmium blackens tissue samples completely and structures are less recognizable within them. This makes it difficult to search for specific structures within embedded tissue samples. Usually this problem is solved by the cutting and examining of semithin sections. In our investigations on the motor end plates of skeletal muscle fibers, we examined imidazoleosmium postfixation, which provides results as good as normal (Voigt et al., 2002). An essential advantage of the imidazole-osmium postfixation is that the tissue samples become translucently reddish during postfixation, so they can be transilluminated with point light sources. In this study we demonstrate the use of imidazole-osmium postfixation on mouse jejunum.

\section{Materials and Methods}

The investigations were carried on the jejunum, liver, gall bladder, and trachea of mice. Following anaesthesia with a

Received July 17, 2003; accepted April 9, 2004.

*Corresponding author. E-mail: tilman.voigt-gawatz@unifr.ch mixture of Rompun (Bayer), and Ketanest (Parke, Davis \& Company), perfusion of the mice was performed from the left ventricle by prerinsing with Thomaedex 40 (Thomae) and fixation with $2.5 \%$ glutaraldehyde in $0.1 \mathrm{M}$ cacodylate buffer. The tissue was cut to size $(\sim 500-\mu$ m-thick rings at jejunum and trachea, edge length $1 \mathrm{~mm}$ at the liver) and fixed by immersion in $2.5 \%$ glutaraldehyde in $0.1 \mathrm{M}$ cacodylate buffer for $3 \mathrm{~h}$.

These tissue samples were divided into two groups and subjected in parallel to postfixation for $1 \mathrm{~h}$ in (1) $1 \%$ osmium in $0.1 \mathrm{M}$ cacodylate buffer or (2) in $1 \%$ osmium with $0.1 \mathrm{M}$ imidazole in $0.1 \mathrm{M}$ cacodylate buffer.

Following this, all of the samples were washed three times for $5 \mathrm{~min}$ in distilled water and dehydrated in an ascending alcohol series and embedded in EPON.

The blocks were analyzed with magnifying glasses (Wild Heerbrugg Photomakroskop M400) or a light microscope (Zeiss), both in the untrimmed and in the trimmed state. They were photographed via the ocular with a digital camera (Canon PowerShot S40, aperture 8, exposure time $1 \mathrm{~min}$; light sensitivity ISO50, ocular adapter [Leica]).

Semithin sections were stained according to Richardson et al. (1960). Some ultrathin sections were sectionstained for 20 min with a saturated solution of uranyl acetate in distilled water and afterward for $5 \mathrm{~min}$ with lead citrate (for the recipe of lead citrate, see Reynolds, 1963). Unstained sections and the section-stained sections from all tissue samples were examined in the TEM (EM10, Zeiss). 


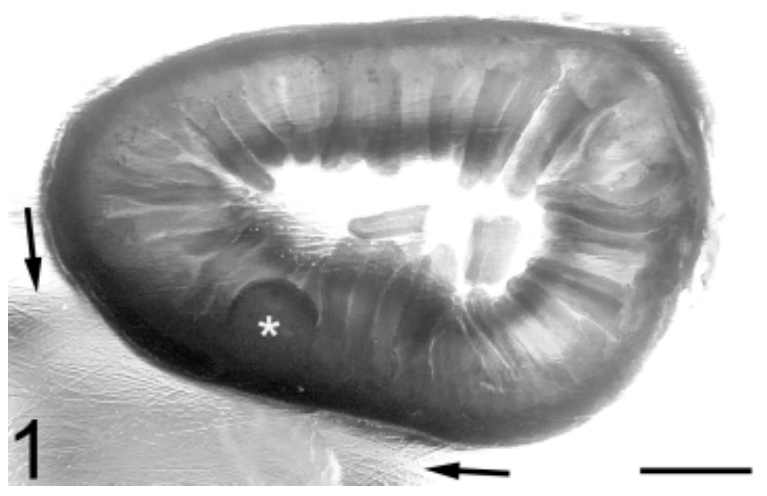

Figure 1. A ring of the jejunum $\sim 500 \mu \mathrm{m}$ thick can be transilluminated easily. A nodulus lymphaticus solitarius can be shown below the tunica muscularis $\left(^{*}\right)$. A specimen label in the block throws its shadow onto the tissue sample (arrows). Untrimmed block before the cutting of semithin sections; imidazole-osmium postfixation; bar $=500 \mu \mathrm{m}$.

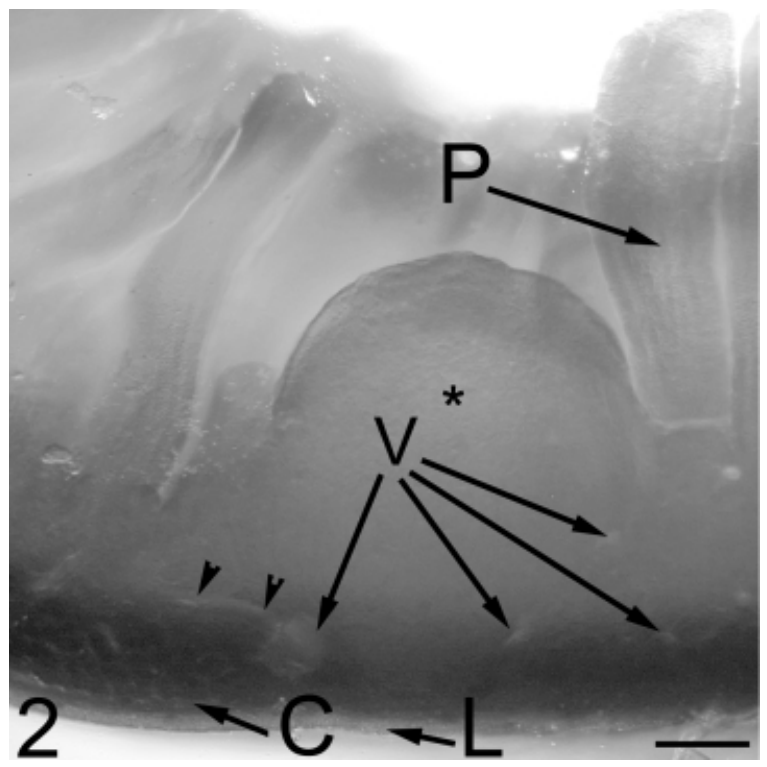

Figure 2. Detail of the untrimmed transilluminated block of Figure 1. Vessels (V) as well as the lamina propria of the villi (P) can be shown in the embedded tissue sample. The vessel branches can be traced (arrowheads). The circular layer (C) and the longitudinal layer (L) of the tunica muscularis are shown; nodulus lymphaticus solitarius $\left(^{*}\right)$. Untrimmed block before the cutting of semithin sections; imidazole-osmium postfixation; bar $=100 \mu \mathrm{m}$.

\section{Results}

The slightly yellowish osmium solution turns reddish during the mixture with imidazole, as does the fixed tissue. A reddish precipitate that sometimes forms does not disturb the result of the postfixation. After embedding in EPON the

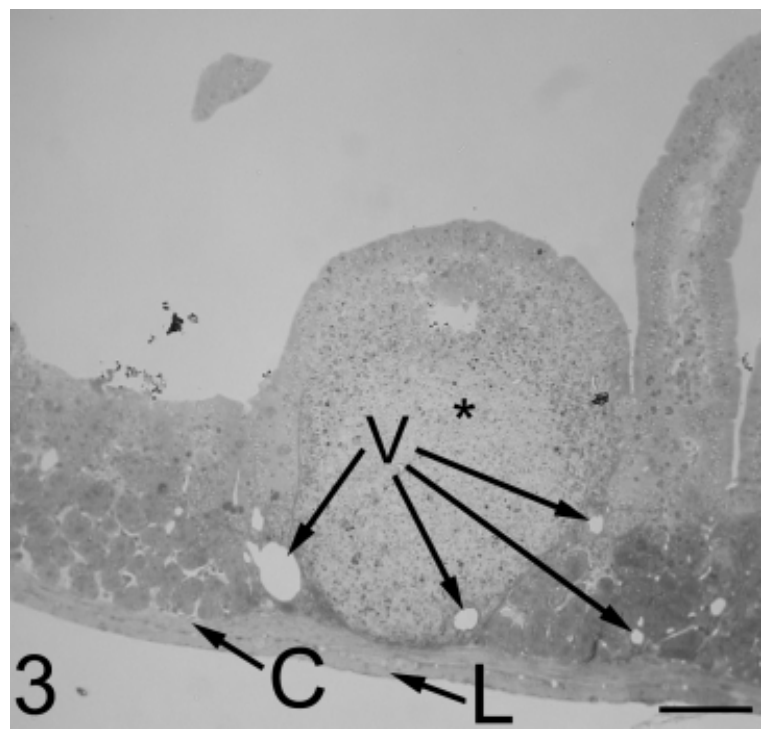

Figure 3. Semithin section $(0.5 \mu \mathrm{m})$ in the area of the nodulus lymphaticus solitarius ${ }^{*}$ ). Vessels (V) that could be seen in the untrimmed block (cf. with Fig. 2) are seen in the semithin section except for the vessel branches; circular layer (C) and longitudinally layer (L) of the tunica muscularis. Imidazole-osmium postfixation; bar $=100 \mu \mathrm{m}$.

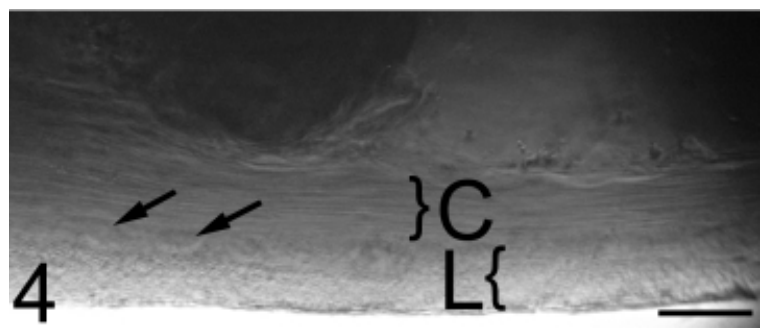

Figure 4. Detail of the block of Figure 1 after cutting of the semithin section in Figure 3. The circular layer (C) and the longitudinal layer (L) of the tunica muscularis can be seen. Ganglion cells of the plexus myentericus can be shown between these two layers (arrows). Trimmed block after the cutting of semithin sections; imidazole-osmium postfixation; bar $=25 \mu \mathrm{m}$.

imidazole-osmium postfixed tissue remains reddish and can be transilluminated with point light sources (Fig. 1).

After our experiences, the following statements, with respect to the transillumination, the section staining, and the contrast, also apply to the liver, gall bladder, and trachea. But the representation is limited to the jejunum because its morphology is ideal to represent the consequences this form of transillumination could have for the handling of embedded tissue samples.

The layers of jejunum, that is, tunica mucosa, tela submucosa, and the tunica muscularis (Figs. 2 and 4 ) as well as noduli lymphatici solitarii with their reaction centers (Figs. 1-3) or the plexus myentericus (Fig. 4) can be recog- 


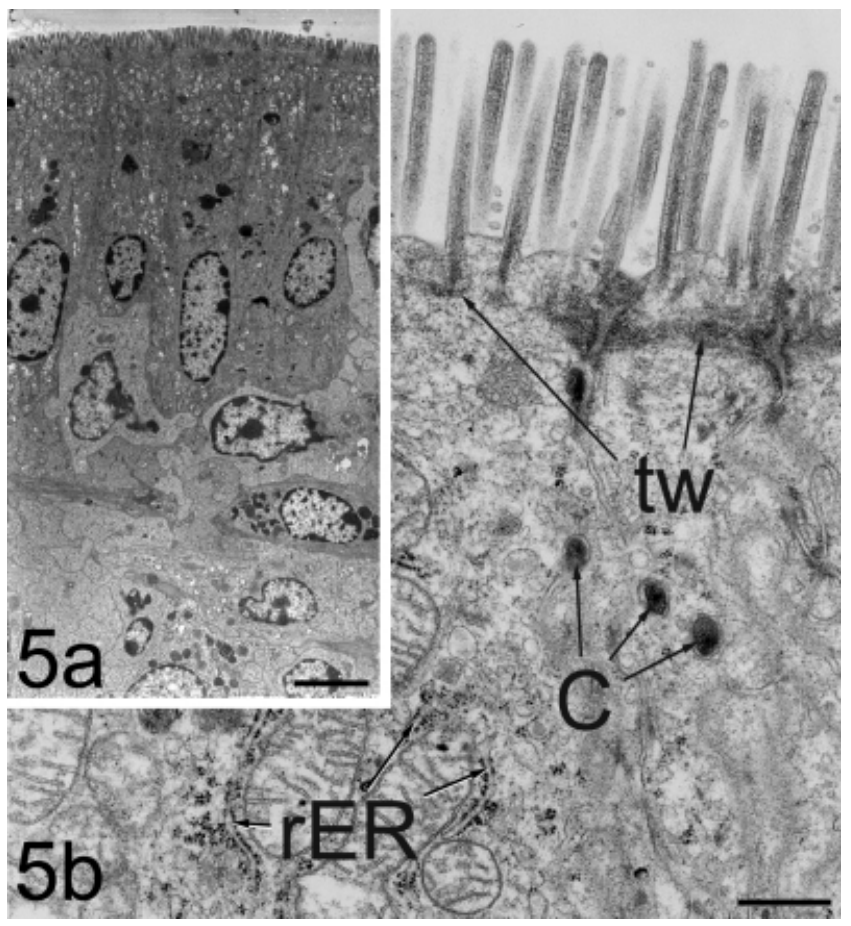

Figure 5. a: Overview of the tunica mucosae with epithelium cells and lamina propria. b: Detail of epithelium cells of the tunica mucosae; terminal web (tw); chylomicrons (C); rough endoplasmic reticulum (rER). Osmium postfixation, section staining with uranyl acetate and lead citrate; bar $=5 \mu \mathrm{m}(\mathbf{a})$, bar $=500 \mathrm{~nm}(\mathbf{b})$.

nized. Also blood vessels and their ramifications in the embedded tissue sample can be traced by focusing with the focusing wheel of the light microscope (cf. Figs. 2 and 3).

But the jejunum sample turns black during the osmium postfixation. So in spite of identical thicknesses, the osmium postfixed jejunum can no longer be transilluminated (not shown). In the most favorable case, blackly colored villi can be recognized in the lumen of the jejunum (not shown).

Sections of tissue postfixed by osmium without imidazole must be subjected first to staining with uranyl acetate and lead citrate (Fig. 5a,b) to achieve sufficient contrast in the TEM.

But sections of imidazole-osmium postfixed tissue can be examined in the TEM without section staining with uranyl acetate and lead citrate (Fig. 6a,b). All membranes of the cells as well as lipid droplets and chylomicrons are stained. But protein-containing structures such as ribosomes, heterochromatin, and the cytoplasma stay unstained. If the sections are stained with uranyl acetate and lead citrate, also ribosomes, the heterochromatin, and the cytoplasma are represented well (Fig. 7a,b).

By comparing the section-stained sections of both differently postfixed tissue samples, it becomes apparent that the chylomicrons as well as the membranes of the imidazole-

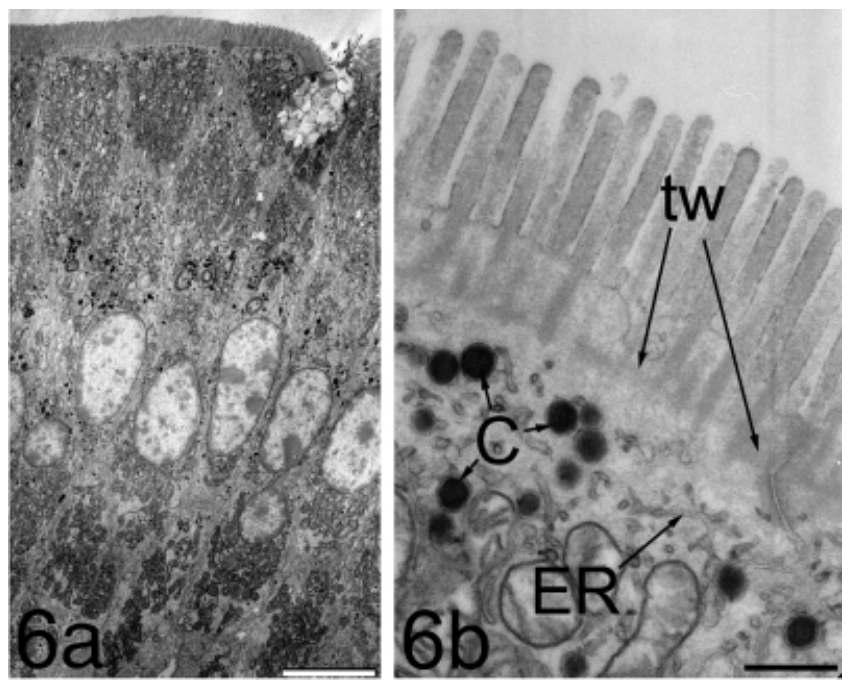

Figure 6. a: Overview of the tunica mucosae with epithelium cells and lamina propria. $\mathbf{b}$ : Detail of epithelium cells of the tunica mucosae; chylomicrons (C); endoplasmic reticulum (ER), terminal web (tw). Imidazole-osmium postfixation, unstained; bar $=5 \mu \mathrm{m}$ (a), bar $=500 \mathrm{~nm}(\mathbf{b})$.
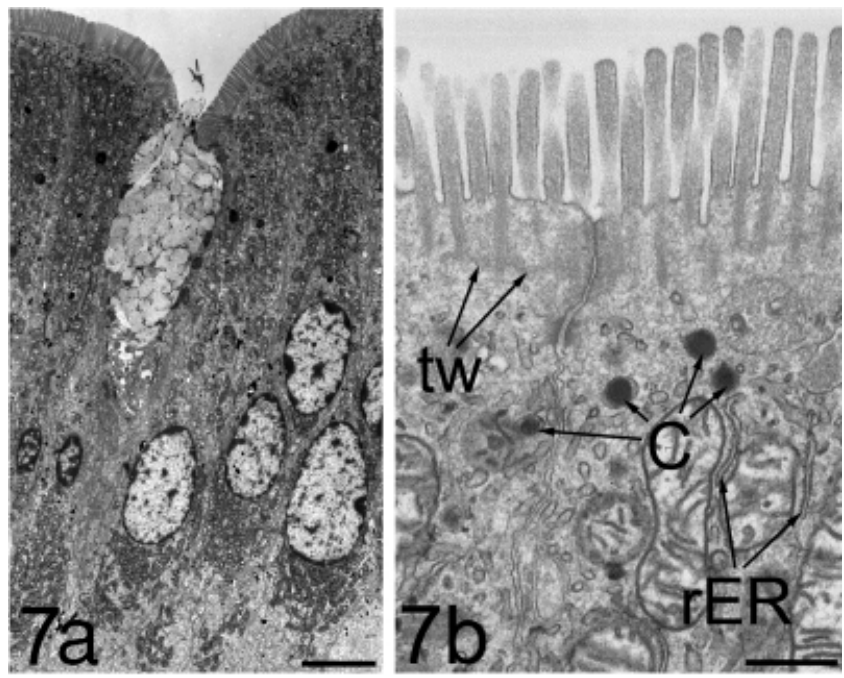

Figure 7. a: Overview of the tunica mucosae with epithelium cells and lamina propria. b: Detail of epithelium cells of the tunica mucosae; chylomicrons (C); rough endoplasmic reticulum (rER), terminal web (tw). Imidazole-osmium postfixation, section staining with uranyl acetate and lead citrate; bar $=5 \mu \mathrm{m}(\mathbf{a})$, bar $=$ $500 \mathrm{~nm}$ (b).

osmium postfixed jejunum are stained more intensively (cf. Figs. $7 \mathrm{~b}$ and $5 b)$.

Although the difference in the staining of the ribosomes and the heterochromatin is small, the microvilli and the terminal web of the osmium-postfixed jejunum are stained by the section staining more intensively (compare Figs. $7 b$ and $5 b)$. 


\section{Discussion}

Imidazole-osmium postfixation is a method easily applied by the addition of imidazole to the osmium. Because imidazole dissolves immediately in aqueous solutions there are no complications in the preparation of the solution.

The advantages of the imidazole-osmium postfixation for investigations in the TEM are described elsewhere (Angermüller \& Fahimi, 1982; Voigt et al., 2002, 2003). One advantage is that lipid-containing structures (membranes, lipid droplets) are better stained by this postfixation in the liver (Angermüller \& Fahimi, 1982), in odontoblasts and cells of the enamel organs (Goldberg \& Septier, 1984), in the kidney (Thiéry et al., 1995), and skeletal muscle fibers (Voigt et al., 2002). Another advantage is that the investigation of thick sections in the TEM is facilitated by the improvement in contrast (Voigt et al., 2003). Finally the unstained section can be analyzed in the TEM, so that much work can be saved in the processing of ultrathin sections (Voigt et al., 2002). Also in the jejunum, the cell membranes as well as lipid droplets and chylomicrons are stained more strongly in the imidazole-osmium postfixed samples, so that investigations can be done without further staining of ultrathin sections. If desired, the contrast of proteincontaining structures such as ribosomes can be increased by section staining.

Transillumination of embedded tissue samples can be a further advantage during presectioning processing. The blackening of the tissue samples after the osmium postfixation makes it impossible to recognize details in the embedded sample. But histological details can be recognized in the block with transillumination of imidazol-osmium postfixed samples, which allows preselection of blocks. After section- ing, the surface of the block is polished. Through that the tissue samples can show finer details, such as the stratification of the tunica muscularis and the plexus myentericus (Fig. 4). These recognized structures can be useful for orientation in the section during investigation with the TEM.

\section{RefERENCES}

Angermüller, S. \& Fahimi, H.D. (1982). Imidazole-buffered osmium tetroxide: An excellent stain for visualization of lipids in transmission electron microscopy. Histochem J 14, 823-835.

Goldberg, M. \& Septier, D. (1984). Demonstration of lipids by imidazole-buffered osmium tetroxide in the odontoblasts and cells of the enamel organ in the rat incisor. J Biol Buccale 12, 317-330.

Reynolds, E.S. (1963). The use of lead citrate at high $\mathrm{pH}$ as an electron-opaque stain in electron microscopy. J Cell Biol 17, 208-212.

Richardson, K.C., Jarett, L. \& Finke, E.H. (1960). Embedding in epoxy resins for ultrathin sectioning in electron microscopy. Stain Technol 35, 313-323.

Thiéry, G., Bernier, J. \& Bergeron, M. (1995). A simple technique for staining of cell membranes with imidazole and osmium tetroxide. J Histochem Cytochem 43, 1079-1084.

Voigt, T., Dauber, W., Bensemann-Ryvkin, I. \& Härtel, X. (2002). Increasing membrane contrast by means of imidazoleosmium post-fixation as exemplified by skeletal muscle fiber. Microsc Res Technol 58, 121-124.

Voigt, T., Dauber, W., Bensemann-Ryvkin, I. \& Härtel, X. (2003). The shape and the position of the sarcoplasmic reticulum and the Golgi apparatus in the sole plate and the remaining subsarcolemmal muscle region of the mouse using imidazole-osmium staining. Microsc Res Technol 61, 419-422. 